\title{
Genome-wide association study and in silico functional analysis of the number of embryos produced by Holstein donors
}

\author{
C. Jaton, ${ }^{*} \dagger^{1}$ F. S. Schenkel, ${ }^{*}$ M. Sargolzaei, ${ }^{*} \dagger$ A. Cánova, ${ }^{*}$ F. Malchiodi, ${ }^{*}$ C. A. Price,$\ddagger$ C. Baes, ${ }^{*}$ \\ and F. Miglior*\$ \\ *Centre for Genetic Improvement of Livestock (CGIL), Department of Animal Biosciences, University of Guelph, Guelph, Ontario, \\ Canada, N1G 2W1 \\ †The Semex Alliance, Guelph, Ontario, Canada, N1G $3 Z 2$ \\ ¥Faculté de Médecine Vétérinaire, Université de Montréal, St-Hyacinthe, Québec, Canada, J2S 2M2 \\ §Canadian Dairy Network (CDN), Guelph, Ontario, Canada, N1K 1E5
}

\begin{abstract}
Superovulation or ovum pick-up and in vitro fertilization are technologies used to produce an increased number of embryos from elite females. Embryo production traits have been shown to be heritable, but the genes that cause this variability have not yet been assessed. The main objectives of this study were to perform a genome-wide association study (GWAS) to find single nucleotide polymorphisms (SNP) associated with embryo production traits and to identify candidate genes affecting the number of embryos produced by Holstein donors in Canada that may provide insight into the regulation of embryo production. Breeding values were estimated and de-regressed for all donors and sires using a data set of 150,971 records of superovulation or ovum pick-up and in vitro fertilization. A total of 11,607 animals were genotyped, but of that number only 5,118 were genotyped with at least a $50 \mathrm{~K}$ SNP panel and had a de-regressed estimated breeding value reliability of at least 10\%. For the GWAS, 606,406 imputed SNP on 29 autosomal chromosomes were considered after applying quality control measures. A singleSNP univariate mixed linear animal model was used to perform the GWAS, and a $5 \%$ false discovery rate was applied to adjust for multiple testing. We found 36 and 14 significant SNP associated with the total number of embryos and the number of viable embryos, respectively, with most of them located on chromosome 11. Using these significant SNP, positional genes located within 10,000 bp upstream and downstream of the SNP were retrieved. Thirteen genes were harboring or near the significant SNP for the total number of embryos, 4 of them also being near the significant SNP for vi-
\end{abstract}

Received September 18, 2017.

Accepted April 5, 2018.

${ }^{1}$ Corresponding author: cjaton@semex.com or cjaton@uoguelph.ca able embryos. Some of these genes (CRB2, DENND1A, MAD1L1, NDUFA8, PTGS1) could be considered as potential positional candidate genes related to the number of embryos produced by a donor. This list will need to be validated in an independent population to confirm the role of the genes for embryo production.

Key words: GWAS, candidate gene, embryo production, Holstein

\section{INTRODUCTION}

Assisted reproductive technologies, such as superovulation and ovum pick-up and in vitro production (OPU-IVP) of embryos, are frequently used in the Canadian dairy industry to produce more offspring from elite donor cows. Although technical improvement of these procedures has been the subject of much research (Mapletoft and Bó, 2011), there is evidence that genetic improvements may also be possible. The number of embryos produced by a donor is moderately heritable in Holstein dairy cattle (Jaton et al., 2016a; Cornelissen et al., 2017; Parker Gaddis et al., 2017), so that it could be possible to genetically select donors that would produce more embryos. Moreover, finding genes that affect embryo production traits may also help with the selection of donors that respond well to superovulation or OPU-IVP. Genome-wide association studies (GWAS) have been performed for fertility traits in dairy (Kolbehdari et al., 2008; Huang et al., 2010; Berry et al., 2012; Cochran et al., 2013; Minozzi et al., 2013; Parker Gaddis et al., 2016) and beef cattle (McDaneld et al., 2014); however, very few studies have performed a GWAS for traits related to embryo production using a large data set. Some studies have used a candidate gene approach to investigate the association of specific genes and embryo production traits in Holstein donors. Some significant SNP on the follicle-stimulating hormone receptor $(F S H R)$ gene were found to be associated with the total number of ova and the number of 
transferable embryos produced by donors (Yang et al., 2010). Another study indicated that some SNP in the FSHR gene could help to identify donors that produce many or few embryos (Cory et al., 2013). Significant SNP on the gonadotropin-releasing hormone receptor (GNRHR) gene were also found to be significantly associated with the number of degenerated embryos, the number of transferable embryos, and the total number of ova produced by Holstein donors (Yang et al., 2011). One SNP located in the insulin-like growth factor 1 receptor (IGFR1) gene was significantly associated with the total number of ova (Yang et al., 2013). Similarly, $1 \mathrm{SNP}$ on the glutamate receptor AMPA 1 (GRIA1) gene was reported to influence the number of ova and embryos collected in superovulated donors (Sugimoto et al., 2010). However, in all cases, few donors were used, which constitutes an important limitation.

The main objectives of our study were to perform a GWAS to find SNP associated with embryo production traits using a large data set of 5,118 genotyped animals and to identify potential key regulator genes affecting the number of embryos produced and to retrieve the biological pathways and mechanisms linked to the number of embryos produced. This study should provide insight into the regulation of embryo production.

\section{MATERIALS AND METHODS}

\section{Data}

A data set containing all superovulation or OPU-IVP procedures performed in Canada over the last 35 years was provided by Holstein Canada (Brantford, ON; www .holstein.ca). This data set was used in our previous studies on genetic analysis of superovulatory response of Holstein cows (Jaton et al., 2016a,b) and the same edits were performed. After editing, 150,971 records from 59,586 donors were considered for the analysis, with 1 record corresponding to 1 superovulation or OPU-IVP procedure performed on 1 donor.

Traits. The 2 embryo production traits that were analyzed from the data set provided were the total number of embryos (NE) and the number of viable embryos (VE) per procedure. The difference between these traits is that VE does not include degenerated or dead embryos recovered.

Pedigree. An animal pedigree file containing 32,587 animals was generated by tracing the pedigrees of the genotyped donors back to 1950.

$\boldsymbol{E} \boldsymbol{B} \boldsymbol{V}$. Genetic parameters and EBV for both embryo traits were estimated from univariate analyses, as described by Jaton et al. (2016a). Breeding values from natural logarithmic transformation were considered for the GWAS. Overall, 63,482 donors and their sires had EBV available for both traits. The EBV were deregressed using a simplified method for de-regression (VanRaden and Sullivan, 2010). Animals with a reliability of de-regressed EBV lower than $10 \%$ for both traits were not considered for further analysis.

Genotypes and Imputation. Genotypes were available for 11,607 donors and sires that had de-regressed EBV. Of that number, 6,734 animals were genotyped with at least a 50K SNP panel, and these genotypes were imputed to high-density genotypes using the FImpute software (Sargolzaei et al., 2014) and a reference population of 2,653 high-density genotypes. After accounting for the reliability threshold, 5,118 individuals (905 males and 4,213 females) were considered for further analyses of the $\mathrm{NE}$ and the $\mathrm{VE}$, respectively.

\section{GWAS}

Quality Control. Quality control analysis was performed including the exclusion of SNP having a minor allele frequency lower than $1 \%$, a call rate lower than $90 \%$, an excess of heterozygosity higher than $15 \%$, and Mendelian error frequency larger than 5\%. The SNP that were out of Hardy-Weinberg equilibrium with very low probability $\left(1 \times 10^{-8}\right)$ and individuals with a call rate lower than $90 \%$ were also excluded. Parentage verification was performed on genotyped parent-progeny pairs, and we observed no parentage conflicts. Overall, 734,077 SNP on 29 autosomal chromosomes were considered for the association analysis before applying quality control measures.

Method. A single SNP univariate mixed linear animal model was used to perform the GWAS using the snp1101 software version 1.0 (Sargolzaei, 2014). Considering the large variation in the reliabilities of the de-regressed EBV, the mixed model equations were weighted by reliabilities. To account for population structure, a random animal effect was fit in the model with (co)variance structure based on the genomic relationship matrix $(\mathbf{G})$. The model was

$$
\mathbf{y}=\mu+\mathbf{W b}+\mathbf{Z g}+\mathbf{e}
$$

where $\mathbf{y}$ is a vector of de-regressed EBV for either NE or $\mathrm{VE} ; \mu$ is the overall mean; $\mathbf{b}$ is a vector of fixed effects including the SNP effect; $\mathbf{g}$ is a vector of random animal effects; $\mathbf{e}$ is a vector of random residuals; and $\mathbf{W}$ and $\mathbf{Z}$ are the corresponding incidence matrices relating $\mathbf{y}$ to $\mathbf{b}$ and $\mathbf{g}$, respectively.

Random effects were assumed to be normally distributed, with means equal to zero and covariance structure equal to 


$$
\operatorname{Var}\left[\begin{array}{l}
g \\
e
\end{array}\right]=\left[\begin{array}{cc}
\mathbf{G} \sigma_{g}^{2} & 0 \\
0 & \mathbf{I} \sigma_{e}^{2}
\end{array}\right],
$$

where $\mathbf{G}$ and $\mathbf{I}$ are the genomic relationship and identity matrices, respectively; and $\sigma_{g}^{2}$ and $\sigma_{e}^{2}$ are the additive genetic and residual variances, respectively.

The quantile-quantile (Q-Q) plot and the inflation factors $(\boldsymbol{\lambda})$ were used to assess the distribution of expected and observed SNP $P$-values. The Q-Q plot is a common graphical tool to examine bias in test statistics, which is often due to underlying population structure; $\lambda$ is calculated as the median or mean of the $\chi^{2}$ test statistics divided by its theoretical median or mean under the null distribution (Devlin and Roeder, 1999). The expected value of $\lambda$ is 1 , which indicates no bias in test statistics.

Correction for Multiple Testing. To adjust for multiple testing, false discovery rate (FDR) at the genome-wise level was controlled at 5\% (Benjamini and Hochberg, 1995). The SNP were considered to be significantly associated with the traits if they were above the 5\% FDR significance level.

Linkage Disequilibrium. Pairwise linkage disequilibrium (LD) of significant SNP was also estimated using snp1101 software to assess whether close markers were gathering the effect of the same or of different genes. The LD was measured using $\mathrm{r}^{2}$, which corresponds to the squared correlation of the alleles at 2 loci (Sargolzaei et al., 2008). The SNP were grouped together if strong LD was found $\left(\mathrm{r}^{2}>0.80\right)$, as tightly linked markers might provide redundant information (Sargolzaei et al., 2008).

SNP Additive Genetic Variance. The expected proportion of the total additive genetic variance explained by each SNP (VAR) was calculated as

$$
\mathrm{VAR}=2 p q \alpha^{2},
$$

where $p$ and $q$ are the observed allele frequencies of the 2 alleles of a given SNP and $\alpha$ is the corresponding estimated allele substitution effect (Falconer and Mackay, 1996).

\section{In Silico Functional Analysis: Gene Ontology Analysis}

Significant SNP at 5\% FDR were mapped to the nearby genes using the bovine genome assembly UMD3.1 (release 84; http://ensembl.org/Bos_taurus/ Info/Index). Genes harboring the significant SNP or located within a distance of 10,000 bp upstream and downstream of the SNP were considered as a starting list for the functional analysis.

Gene ontology (GO) analysis was performed, taking into account the $3 \mathrm{GO}$ categories (biological process, molecular function, and cellular component) using Blast2GO software version 5 (Götz et al., 2008). Bos taurus coding sequences were downloaded from the ENSEMBL biomart martview (http://www.ensembl .org/biomart/martview) and were annotated with Blastx and the Blast2GO mapping and GO annotation routines (Götz et al., 2008). The Blastx algorithm was used to compare a nucleotide query sequence against all the nucleotide sequence database by communicating directly to NCBI (National Center for Biotechnology Information, Bethesda, MD). A statistical significance threshold of 1.0 E-3 (e-value) was established for reporting matches against the database sequences. Also, a minimal length (300 bp) of a blast hit and $90 \%$ of similarity was used to exclude hits with only small local alignments from the blast results. Gene ontology significance levels were computed following Fisher's exact test for multiple testing in Blast2GO comparing the list of positional candidate genes vs the functions from the reference (background genes) using the bovine genome assembly UMD3.1 (release 91). Blast2GO was also used to examine the associated metabolic pathways involved in the list of genes harboring the SNP or within a distance of 10,000 bp upstream and downstream using the Kyoto Encyclopedia of Genes and Genomes (KEGG) to identify the metabolic pathways involved for the significant enzymes found (Cánovas et al., 2013; Li et al., 2016).

\section{RESULTS AND DISCUSSION}

\section{GWAS}

After accounting for quality control criteria, 606,406 SNP were considered for NE and VE. The Q-Q plots are presented for both traits in Figure 1. The values of $\lambda$ for both traits suggested no bias in test statistics. After accounting for multiple comparisons, a total of 36 and 14 SNP were significantly associated at the $5 \%$ FDR level with NE and VE, respectively. Figures 2 and 3 show the distribution of the significant SNP for embryo production traits across the 29 bovine chromosomes. For both traits, we observed a major peak on chromosome 11, and the majority of the significant SNP were located on this chromosome.

For NE, 26 of the significant SNP (26 out of 36) were located on chromosome 11, whereas the other significant SNP were located on chromosomes 2 (3 out of 36), 25 (3 out of 36 ), and 29 (4 out of 36). For 


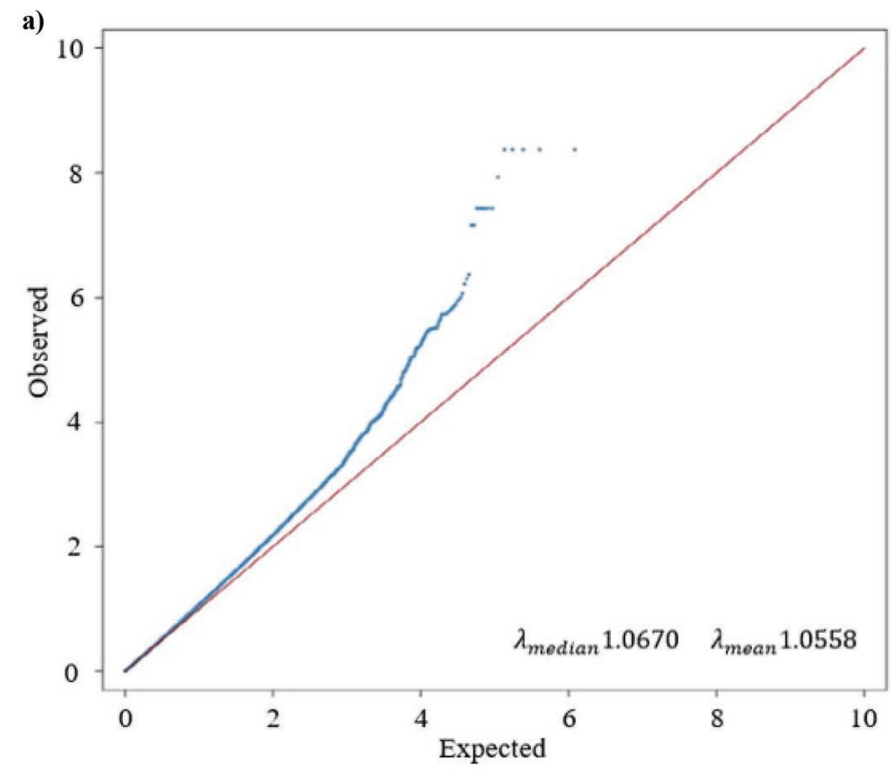

b)

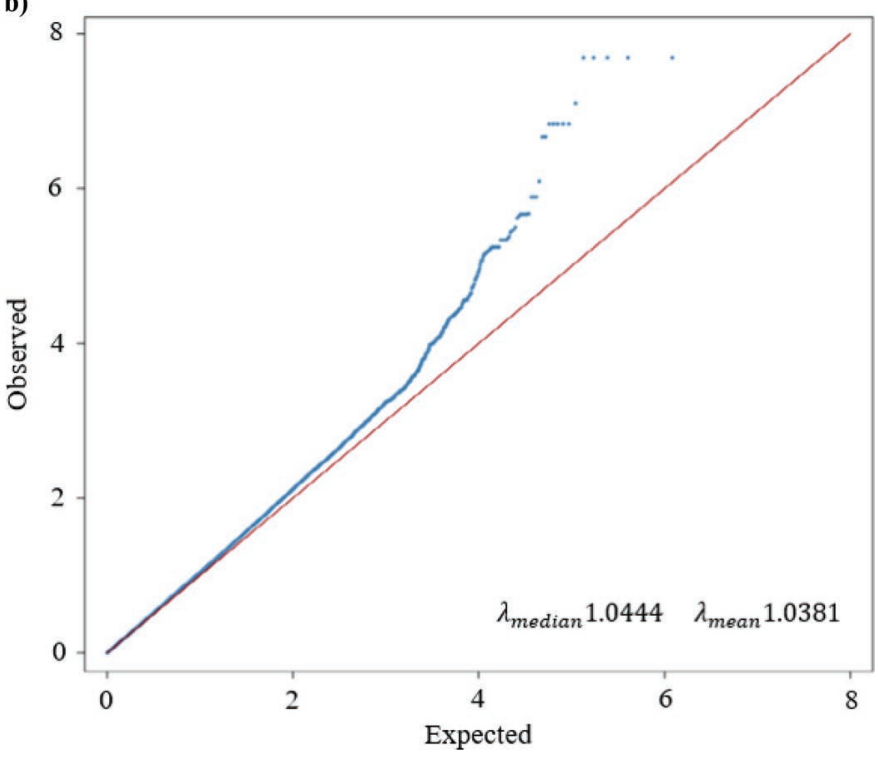

Figure 1. Quantile-quantile plot for (a) total number of embryos and (b) number of viable embryos. $\lambda=$ inflation factor; $\lambda$ is calculated as the median or mean of the $\chi^{2}$ test statistics divided by its theoretical median or mean under the null distribution. Color version available online.

VE, all 14 significant SNP were located on chromosome 11. All 14 SNP had a pleiotropic effect on NE and VE. This was expected, as the 2 traits considered in our study were highly genetically correlated, as reported in a previous study (Jaton et al., 2016a). For both traits, SNP in strong LD were grouped together because they likely capture the effect of the same gene. All significant groups of SNP for NE and VE are listed in Tables 1 and 2, respectively, along with their level of LD. These tables also present the proportion of addi- tive genetic variance explained by the most significant SNP within a group, with the largest value about 1 and $0.5 \%$ for NE and VE, respectively. For all groups, the total proportion of additive genetic variance explained by the groups was about 7 and $1.5 \%$ for $\mathrm{NE}$ and $\mathrm{VE}$, respectively. This shows that only a very small proportion of the total additive genetic variance could be explained by a single SNP or group, demonstrating the likely polygenic nature of the embryo production traits.

To date, very few GWAS have been carried out on embryo production traits, but recently a group from the United States used a single-step approach to identify genomic regions that could explain additive genetic variance for various traits related to embryo production, such as the number of good embryos (Parker Gaddis et al., 2017). Using a 10-SNP window approach, Parker Gaddis et al. (2017) identified regions explaining the most additive genetic variance for total number of structures recovered from superovulation on chromosomes 5 , $8,13,14$, and 21. Similarly, for number of good embryos collected, chromosomes 5, 8, 10, 13, and 14 had regions explaining the largest amount of additive genetic variance. The largest proportion of total additive variance explained by a single 10-SNP window was $0.43 \%$ for the number of good embryos, which is in line with the results of the current study. The main region identified by Parker Gaddis et al. (2017) for both superovulation traits was on chromosome 8 , and some nearby genes, such as NPR2, TLE4, and TGFBR1, were mentioned. One SNP on bovine chromosome $11(89,371,911$ bp) was reported as having a large standardized SNP effect on total number of structures recovered. This SNP is located at $3,546 \mathrm{k}$ bp of one of our significant SNP, which makes unlikely that these SNP are in LD with the same causative mutations. Overall, Parker Gaddis et al. (2017) had only a limited number of records. For the number of structures recovered and the number of good embryos from the superovulation data set, only 926 records from 723 donors were used, and all of these records were from 1 herd. This could partly explain why different regions were found compared with our study.

Considering that embryo production is a fertility trait, GWAS of other fertility traits in Holstein (Kolbehdari et al., 2008; Huang et al., 2010; Berry et al., 2012; Minozzi et al., 2013; Parker Gaddis et al., 2016) and beef (McDaneld et al., 2014) cattle were reviewed to assess if common significant SNP could be found between fertility traits and embryo traits. No common SNP were significant between fertility traits, such as daughter fertility, fertilization rate, 56-d nonreturn rate, number of services, pregnancy rate to first service, and so on, and embryo traits. Moreover, none of the GWAS done for dairy cattle fertility traits have shown a significant 
peak in the same region as our most significant peak on chromosome 11. This could indicate that the number of embryos produced by Holstein donors and other fertility traits of Holstein females are possibly regulated by different genes.

From the GWAS analysis, we identified significant SNP for both NE and VE traits. Genes harboring or near $(\leq 10,000 \mathrm{bp})$ those SNP were listed for NE and $\mathrm{VE}$, to be used in in silico functional analysis. For NE, 13 genes were found harboring or near $(\leq 10,000 \mathrm{bp})$ the significant SNP and 4 of them were also found for VE (Tables 1 and 2).

\section{In Silico Functional Analysis}

The coding sequences for all genes that harbored significant SNP or were within a distance of $10,000 \mathrm{bp}$ upstream and downstream from the significant SNP were downloaded from ENSEMBL biomart martview application. Considering that all genes found for $\mathrm{VE}$ were also found for NE, the analysis was only performed for NE. Nine genes had 1 coding sequence, 1 gene had no coding sequence available (5S_rRNA), and 2 genes had 2 coding sequences (CRB2), so that 13 cod- ing sequences were submitted to Blast2Go for NE. The coding sequence of MORN5 gene was unsuccessfully mapped in Blast2Go, so 12 coding sequences were used to retrieve GO terms associated with NE. Significant GO terms were identified overlapping in at least 7 out of 12 positional candidate genes analyzed [biological process (7 genes), molecular function (8 genes), cellular component (12 genes)], including GO terms associated with biological process involved in the intracellular estrogen signaling pathway, histone citrullination, and chromatin-mediated maintenance of transcription. In addition, combining some interesting GO terms found associated with the list of positional candidate genes together with information from other studies would help to understand how these identified genes could affect embryo production.

The crumbs 2 (CRB2) gene is associated with embryo development, cell differentiation, and anatomical structure formation involved in morphogenesis. In mice, CRB2 has been found to affect the early stages of embryogenesis, considering that CRB2 knockout mice died before embryonic d 12.5 in mice and that by $\mathrm{d} 7.75$ the CRB2 deficient embryos were showing some developmental anomalies compared with embryos that were

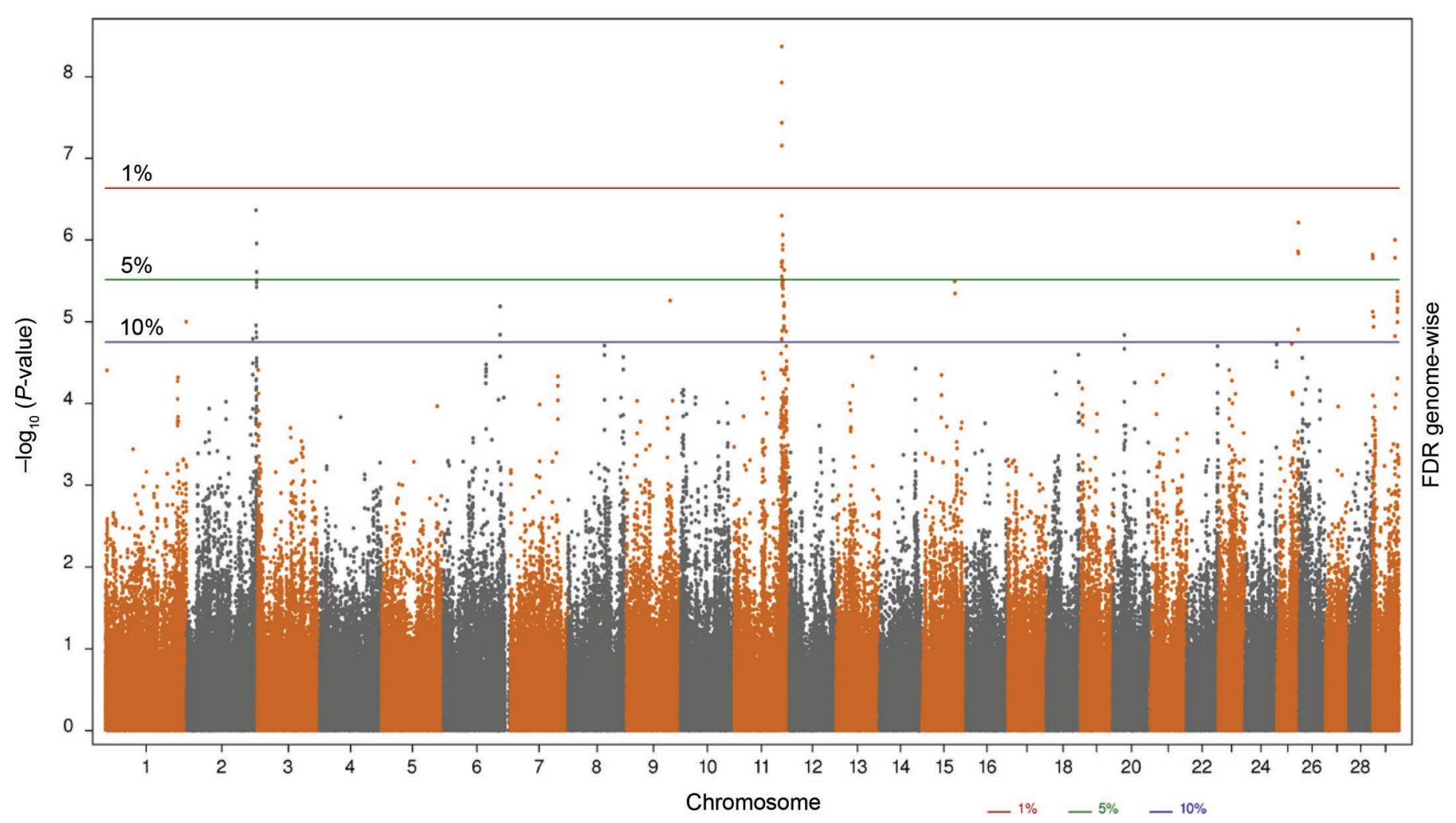

Figure 2. Manhattan plot for total number of embryos with false discovery rate (FDR) controlled at $1 \%$ (red), $5 \%$ (green) and $10 \%$ (blue). Color version available online. 


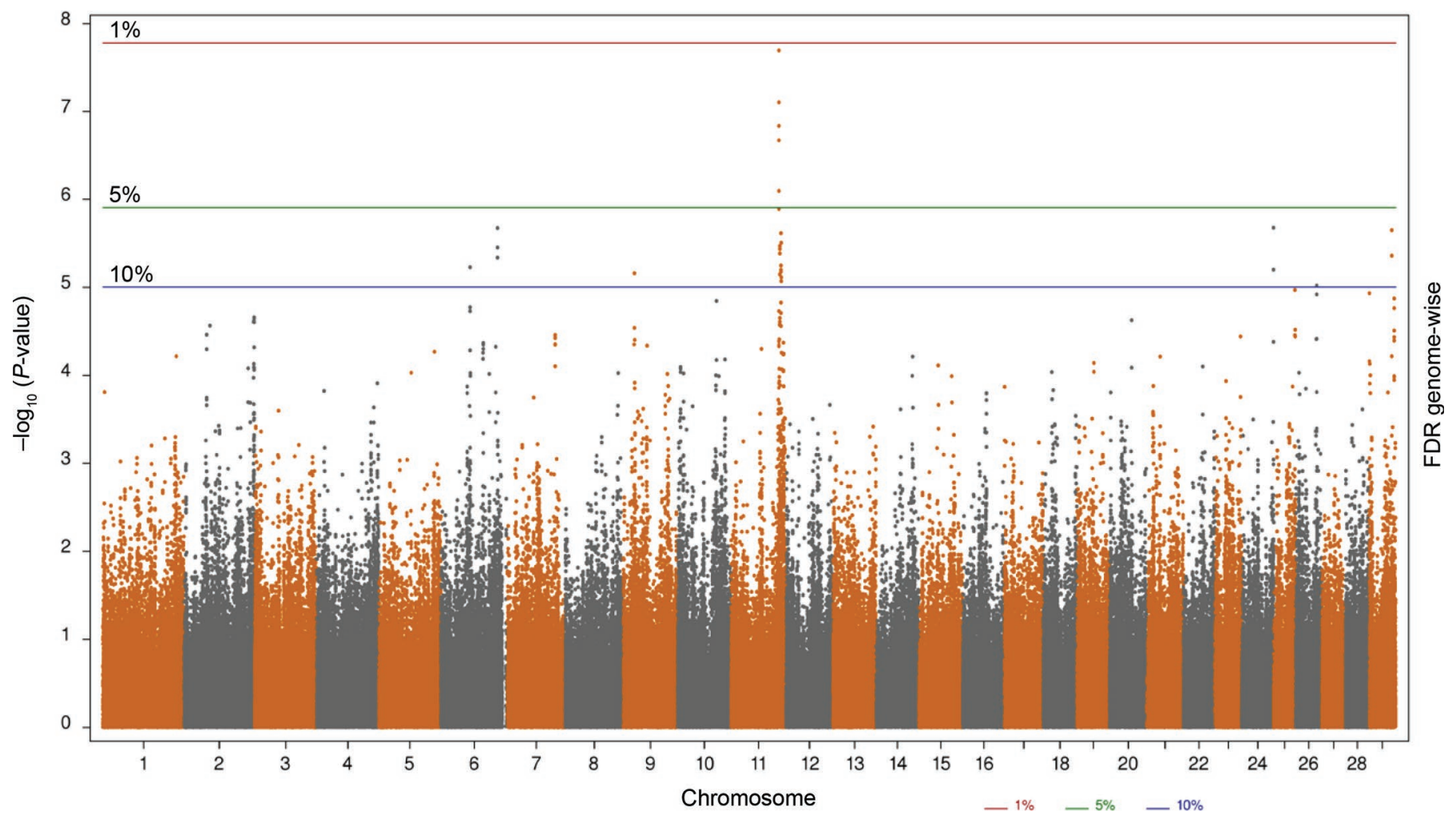

Figure 3. Manhattan plot for number of viable embryos with false discovery rate (FDR) controlled at $1 \%$ (red), $5 \%$ (green) and $10 \%$ (blue). Color version available online.

expressing the gene (Xiao et al., 2011). The role this protein may play in embryogenesis in cattle or other species has not been explored.
Chromosome organization and segregation and mitotic nuclear division were the GO terms identified for mitotic arrest deficient 1-like 1 (MAD1L1) gene.

Table 1. Significant genomic regions and SNP associated with total number of embryos and genes harboring or near them (within 10k bases)

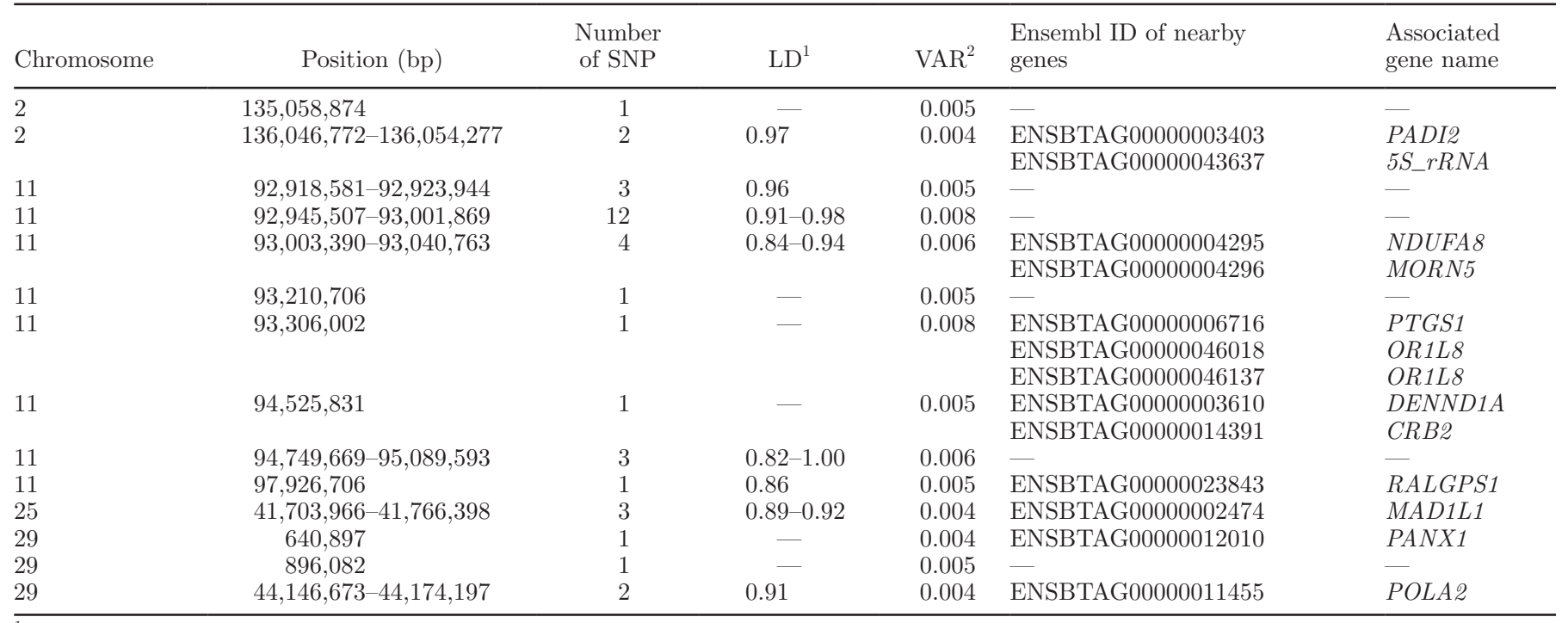

${ }^{1}$ Linkage disequilibrium.

${ }^{2}$ Expected proportion of the total additive genetic variance explained by the most significant SNP in the region. 
Another study performed in mice has shown MAD1 to be present in the oocytes during meiosis, participating namely in spindle checkpoints, which ensures that sister chromatids are aligned before segregation (Zhang et al., 2005).

Gene ontology terms that were found for NDUFA8 gene included generation of precursor metabolites and energy and mitochondrion organization. The NDUFA8 protein is a subunit of NADH dehydrogenase (Triepels et al., 1998), which, as part of the electron transport chain, regulates energy metabolism (Held et al., 2012) and has been associated with decreased developmental competence in oocytes (O'Shea et al., 2012). Protein NDUFA8 is part of the oxidative phosphorylation pathway, which is very important for production of most of the ATP needed for development of the preimplantation embryo (Thompson et al., 1996; St John et al., 2010; Gad et al., 2011). For embryos produced in vitro or in vivo, 92 to $95 \%$ of the ATP is produced by oxidative phosphorylation until the compact morula stage, after which $82 \%$ of ATP is produced by this pathway to support further development (Thompson et al., 1996). Some studies have reported that this pathway is involved in preimplantation development (Held et al., 2012), is significantly associated with fresh bovine embryo produced in vitro (Aksu et al., 2012), and is associated with in vivo-derived blastocysts and slowdividing in vitro-derived blastocysts (Milazzotto et al., 2016). Oxidative phosphorylation was also a dominant pathway for blastocyst-stage embryos produced by superovulated heifers (Gad et al., 2011).

The polymerase $\alpha 2$ accessory subunit (POLA2) gene encodes an enzyme, DNA polymerase, involved in purine and pyrimidine metabolism. To date very little information is available about the association between this DNA remodeling gene and embryo production. However, one study performed in mice reported POLA2 to be a gene specifically expressed in embryonic stem cells and embryonic germ cells (Sharov et al., 2003).

The DENN domain containing 1A (DENND1A) gene encodes a nucleotide exchange factor for the GTPase Rab35, which is involved in endosomal recycling. Gene
DENND1A is overexpressed in the theca cells of women with polycystic ovary syndrome (PCOS), considering that it has been identified in different human populations (McAllister et al., 2014, 2015); PCOS is associated with several reproduction problems, such as anovulation and infertility, and is also characterized by an arrest of follicular development because ovarian theca cells secrete excessive amounts of androgen (McAllister et al., 2014). Gene DENND1A was reported to be highly expressed in PCOS theca cells compared with normal theca cells, supporting its causal role on PCOS (McAllister et al., 2015). In cattle, cystic ovarian follicles (COF) are also well known to affect fertility; however, the level of androgen seems to be not influenced by $\mathrm{COF}$ in cattle (Eyestone and Ax, 1984). No studies have specifically looked at the effect of DENND1A for cattle COF.

Prostaglandin-endoperoxide synthase 1 (PTGS1, also known as COX1), was located at 8,581 bp from one of the significant SNP for both NE and VE. Gene PTGS1 catalyzes the conversion of arachidonic acid into prostaglandin $\mathrm{G}_{2}$, which is then reduced into prostaglandin $\mathrm{H}_{2}$, which is a precursor of various forms of prostaglandins (Needleman et al., 1986; Kanehisa and Goto, 2000; Kanehisa et al., 2016), such as prostaglandin $\mathrm{E}_{2}$ and $\mathrm{PGF}_{2 \alpha}$ (Arosh, 2002; Lee et al., 2007; Li et al., 2012). Prostaglandins play important roles in female reproduction, including luteolysis ovulation, fertilization, implantation, and parturition (Wang et al., 2002; Ball and Peters, 2004; Gauvreau et al., 2010; Saint-Dizier et al., 2011; Kim et al., 2014). Specifically, $\mathrm{PGF}_{2 \alpha}$ is used in superovulation protocols to cause the regression of the corpus luteum and to induce ovulation of the recruited follicles (Ball and Peters, 2004; Mapletoft, 2006; Moore and Thatcher, 2006). It has been presumed that human embryos can synthetize prostaglandins during the preimplantation period through PTGS1 and PTGS2 proteins, with PTGS1 being essentially expressed in the early stage of development and PTGS2 being mainly expressed at the 8-cell stage and later (Wang et al., 2002). Bovine embryos produce prostaglandin in vivo and in vitro (Torres et al., 2015), and high concentrations of $\mathrm{PGF}_{2 \alpha}$ have been reported to have a negative

Table 2. Significant genomic regions and SNP associated with number of viable embryos and genes harboring or near them (within 10k bases)

\begin{tabular}{|c|c|c|c|c|c|c|}
\hline Chromosome & Position (bp) & $\begin{array}{l}\text { Number } \\
\text { of SNP }\end{array}$ & $\mathrm{LD}^{1}$ & $\mathrm{VAR}^{2}$ & $\begin{array}{l}\text { Ensembl ID of nearby } \\
\text { genes }\end{array}$ & $\begin{array}{l}\text { Associated } \\
\text { gene name }\end{array}$ \\
\hline 11 & $92,945,507-93,001,869$ & 12 & $0.91-0.98$ & 0.005 & ENSBTAG00000004295 & NDUFA 8 \\
\hline \multirow[t]{3}{*}{11} & $93,306,002$ & 1 & - & 0.005 & ENSBTAG00000006716 & PTGS1 \\
\hline & & & & & ENSBTAG00000046018 & OR1L8 \\
\hline & & & & & ENSBTAG00000046137 & OR1L8 \\
\hline
\end{tabular}

\footnotetext{
${ }^{1}$ Linkage disequilibrium.

${ }^{2}$ Expected proportion of the total additive genetic variance explained by the most significant SNP in the region.
} 
effect on embryo development (Kim et al., 2014). In dairy cattle, PTGS2 but not PTGS1 was reported to be involved in blastocyst expansion and development of embryos (Saint-Dizier et al., 2011). The PTGS1 gene is constitutively expressed in all tissues and was reported to have more of a housekeeping function (Asselin et al., 1997; Wang et al., 2002) compared with PTGS2. However, other studies have shown that PTGS1 plays a main role in oviduct epithelial cells, which is where fertilization and early embryonic development naturally take place (Gauvreau et al., 2010). Considering that 1 significant SNP was found to be nearby PTGS1 for both $\mathrm{NE}$ and $\mathrm{VE}$, it is possible that the level of prostaglandin produced by this enzyme could affect the developmental competence of oocytes, thereby affecting the number of embryos produced.

Regarding the biological pathways, numerous studies in dairy cattle have shown an association between the number of embryos produced and serum levels of anti-Müllerian hormone (AMH) of the donors (Rico et al., 2009, 2012; Monniaux et al., 2010; Guerreiro et al., 2014; Souza et al., 2015). The AMH is mainly expressed in granulosa cells of small antral follicles (Rico et al., 2009, 2012; Monniaux et al., 2010), and its serum concentration is an indicator of the ovarian follicular reserve (Lie Fong et al., 2008; Guerreiro et al., 2014; Peluso et al., 2014). The AMH modulates the sensitivity of growing follicles to FSH (Lie Fong et al., 2008; Guerreiro et al., 2014), therefore regulating the number of growing follicles that can eventually ovulate (Guerreiro et al., 2014; Peluso et al., 2014; Souza et al., 2015). Plasma AMH concentrations are variable between individuals, but are highly repeatable within individuals (Rico et al., 2012), and are known to be a useful and reliable predictor of the number of embryos produced in vitro (Guerreiro et al., 2014) or in vivo (Rico et al., 2009; Monniaux et al., 2010; Souza et al., 2015). Response to ovarian stimulation in cattle and humans with high AMH concentrations is generally more pronounced (Rico et al., 2009; Souza et al., 2015). Among them, biological pathways associated with AMH were retrieved from KEGG (Kanehisa and Goto, 2000; Kanehisa et al., 2016), namely the cyclic AMP signaling pathway, cytokine-cytokine receptor interaction, transforming growth factor- $\beta$ signaling pathway and Hippo signaling pathway. The cyclic AMP signaling pathway is linked with the olfactory transduction pathway. Considering that we had significant SNP located nearby a gene part of the olfactory family (i.e., OR1L8), it would be interesting to further investigate the link between AMH and embryo production.

Therefore, additional in-depth functional studies, for example including gene expression data, will be necessary to better understand the metabolic and molecular processes involved and the biology underlying the gene regulation and its possible effect on the number of embryos produced by a donor.

\section{CONCLUSIONS}

This GWAS for embryo production traits in Canadian Holstein cattle found significant SNP associated with the total number of embryos and the number of viable embryos produced by a donor. The SNP were mainly located on chromosome 11 for both traits. The GWAS provided a list of genes harboring or nearby the significant SNP, including CRB2, DENND1A, MAD1L1, NDUFA8, and PTGS1. From the in silico functional analysis some interesting GO terms and metabolic pathways were identified for these genes. However, further in-depth functional analysis will be needed to identify the potential regulator genes and the functional SNP associated with embryo production. Therefore, this study provided a list of potential positional candidate genes harboring or near SNP related to the total number of embryos produced by a donor. This list will need to be validated in an independent population to confirm the role of the genes for embryo production.

\section{ACKNOWLEDGMENTS}

The authors are grateful to Holstein Canada (Brantford, ON) for providing the data. This study was funded by CIAQ (St-Hyacinthe, Quebec, Canada), The Semex Alliance (Guelph, Ontario, Canada), the DairyGen Council of Canadian Dairy Network (Guelph, Ontario, Canada) and the Natural Sciences and Engineering Research Council of Canada (Ottawa, Ontario, Canada).

\section{REFERENCES}

Aksu, D. A., C. Agca, S. Aksu, H. Bagis, T. Akkoc, A. T. Caputcu, S. Arat, A. C. Taskin, S. H. Kizil, T. Karasahin, N. Akyol, M. Satilmis, H. Sagirkaya, B. Ustuner, Z. Nur, and Y. Agca. 2012. Gene expression profiles of vitrified in vitro- and in vivo-derived bovine blastocysts. Mol. Reprod. Dev. 79:613-625.

Arosh, J. A. 2002. Expression of cyclooxygenases 1 and 2 and prostaglandin E synthase in bovine endometrial tissue during the estrous cycle. Biol. Reprod. 67:161-169.

Asselin, E., P. Drolet, and M. A. Fortier. 1997. Cellular mechanisms involved during oxytocin-induced prostaglandin F2alpha production in endometrial epithelial cells in vitro: role of cyclooxygenase-2. Endocrinology 138:4798-4805.

Ball, P. J. H., and A. R. Peters. 2004. Reproduction in Cattle. 3rd ed. Blackwell Pub, Oxford, UK.

Benjamini, Y., and Y. Hochberg. 1995. Controlling the false discovery rate: A practical and powerful approach to multiple testing. J. R. Stat. Soc. Ser. B Methodol. 57:289-300.

Berry, D. P., J. W. M. Bastiaansen, R. F. Veerkamp, S. Wijga, E. Wall, B. Berglund, and M. P. L. Calus. 2012. Genome-wide associations for fertility traits in Holstein-Friesian dairy cows using 
data from experimental research herds in four European countries. Animal 6:1206-1215.

Cánovas, A., G. Rincón, A. Islas-Trejo, R. Jimenez-Flores, A. Laubscher, and J. F. Medrano. 2013. RNA sequencing to study gene expression and single nucleotide polymorphism variation associated with citrate content in cow milk. J. Dairy Sci. 96:2637-2648.

Cochran, S. D., J. B. Cole, D. J. Null, and P. J. Hansen. 2013. Discovery of single nucleotide polymorphisms in candidate genes associated with fertility and production traits in Holstein cattle. BMC Genet. 14:49.

Cornelissen, M. A. M. C., E. Mullaart, C. Van der Linde, and H. A. Mulder. 2017. Estimating variance components and breeding values for number of oocytes and number of embryos in dairy cattle using a single-step genomic evaluation. J. Dairy Sci. 100:46984705 .

Cory, A. T., C. A. Price, R. Lefebvre, and M.-F. Palin. 2013. Identification of single nucleotide polymorphisms in the bovine folliclestimulating hormone receptor and effects of genotypes on superovulatory response traits. Anim. Genet. 44:197-201.

Devlin, B., and K. Roeder. 1999. Genomic control for association studies. Biometrics 55:997-1004.

Eyestone, W. H., and R. L. Ax. 1984. A review of ovarian follicular cysts in cows, with comparisons to the condition in women, rats and rabbits. Theriogenology 22:109-125.

Falconer, D. S., and T. F. C. Mackay. 1996. Introduction to Quantitative Genetics. Longman, Essex, UK.

Gad, A., U. Besenfelder, F. Rings, N. Ghanem, D. Salilew-Wondim, M. M. Hossain, D. Tesfaye, P. Lonergan, A. Becker, U. Cinar, K. Schellander, V. Havlicek, and M. Holker. 2011. Effect of reproductive tract environment following controlled ovarian hyperstimulation treatment on embryo development and global transcriptome profile of blastocysts: Implications for animal breeding and human assisted reproduction. Hum. Reprod. 26:1693-1707.

Gauvreau, D., V. Moisan, M. Roy, M. A. Fortier, and J.-F. Bilodeau. 2010. Expression of prostaglandin E synthases in the bovine oviduct. Theriogenology 73:103-111.

Götz, S., J. M. García-Gómez, J. Terol, T. D. Williams, S. H. Nagaraj, M. J. Nueda, M. Robles, M. Talón, J. Dopazo, and A. Conesa. 2008. High-throughput functional annotation and data mining with the Blast2GO suite. Nucleic Acids Res. 36:3420-3435.

Guerreiro, B. M., E. O. S. Batista, L. M. Vieira, M. F. Sá Filho, C. A Rodrigues, A. Castro Netto, C. R. A. Silveira, B. M. Bayeux, E. A. R. Dias, F. M. Monteiro, M. Accorsi, R. N. V. R. Lopes, and P. S. Baruselli. 2014. Plasma anti-mullerian hormone: An endocrine marker for in vitro embryo production from Bos taurus and Bos indicus donors. Domest. Anim. Endocrinol. 49:96-104.

Held, E., D. Salilew-Wondim, M. Linke, U. Zechner, F. Rings, D. Tesfaye, K. Schellander, and M. Hoelker. 2012. Transcriptome fingerprint of bovine 2-cell stage blastomeres is directly correlated with the individual developmental competence of the corresponding sister blastomere. Biol. Reprod. 87:154.

Huang, W., B. W. Kirkpatrick, G. J. M. Rosa, and H. Khatib. 2010. A genome-wide association study using selective DNA pooling identifies candidate markers for fertility in Holstein cattle: Fertility study using selective DNA pooling. Anim. Genet. 41:570-578.

Jaton, C., A. Koeck, M. Sargolzaei, F. Malchiodi, C. A. Price, F. S. Schenkel, and F. Miglior. 2016a. Genetic analysis of superovulatory response of Holstein cows in Canada. J. Dairy Sci. 99:3612-3623.

Jaton, C., A. Koeck, M. Sargolzaei, C. A. Price, C. Baes, F. S. Schenkel, and F. Miglior. 2016b. Short communication: Genetic correlations between number of embryos produced using in vivo and in vitro techniques in heifer and cow donors. J. Dairy Sci. 99:82228226 .

Kanehisa, M., and S. Goto. 2000. KEGG: Kyoto Encyclopedia of Genes and Genomes. Nucleic Acids Res. 28:27-30.

Kanehisa, M., Y. Sato, M. Kawashima, M. Furumichi, and M. Tanabe. 2016. KEGG as a reference resource for gene and protein annotation. Nucleic Acids Res. 44:D457-D462.

Kim, S.-S., J.-I. Bang, M. Fakruzzaman, K.-L. Lee, D.-H. Ko, N. Ghanem, Z. Wang, and I.-K. Kong. 2014. Effects of flunixin meglumine and prostaglandin F $2 \alpha$ treatments on the development and quality of bovine embryos in vitro. Reprod. Domest. Anim. 49:957-963.

Kolbehdari, D., Z. Wang, J. R. Grant, B. Murdoch, A. Prasad, Z. Xiu, E. Marques, P. Stothard, and S. S. Moore. 2008. A whole-genome scan to map quantitative trait loci for conformation and functional traits in Canadian Holstein bulls. J. Dairy Sci. 91:2844-2856.

Lee, C. R., F. G. Bottone, J. M. Krahn, L. Li, H. W. Mohrenweiser, M. E. Cook, R. M. Petrovich, D. A. Bell, T. E. Eling, and D. C. Zeldin. 2007. Identification and functional characterization of polymorphisms in human cyclooxygenase-1 (PTGS1). Pharmacogenet. Genomics 17:145-160.

Li, M., S. Riddle, H. Zhang, A. D'Alessandro, A. Flockton, N. J. Serkova, K. C. Hansen, R. Moldvan, B. A. McKeon, M. Frid, S. Kumar, H. Li, H. Liu, A. Cánovas, J. F. Medrano, M. G. Thomas, D. Iloska, L. Plecita-Hlavata, P. Jezek, S. Pullamsetti, M. A. Fini K. C. El Kasmi, Q. Zhang, and K. R. Stenmark. 2016. Metabolic reprogramming regulates the proliferative and inflammatory phenotype of adventitial fibroblasts in pulmonary hypertension through the transcriptional co-repressor c-terminal binding protein-1. Circulation 134:1105-1121.

Li, X., M. Guo, C. M. McCracken, and C. D. Funk. 2012. Targeted exchange of an expression cassette encoding cyclooxygenase-2 at the Ptgs1 locus. Prostaglandins Other Lipid Mediat. 99:38-44.

Lie Fong, S., E. Baart, E. Martini, I. Schipper, J. Visser, A. Themmen, F. de Jong, B. Fauser, and J. Laven. 2008. Anti-Müllerian hormone: a marker for oocyte quantity, oocyte quality and embryo quality? Reprod. Biomed. Online 16:664-670.

Mapletoft, R. 2006. Bovine embryo transfer. IVIS Rev. Vet. Med., International Veterinary Information Service, Ithaca NY.

Mapletoft, R. J., and G. A. Bó. 2011. The evolution of improved and simplified superovulation protocols in cattle. Reprod. Fertil. Dev. $24: 278$.

McAllister, J. M., R. S. Legro, B. P. Modi, and J. F. Strauss. 2015. Functional genomics of PCOS: From GWAS to molecular mechanisms. Trends Endocrinol. Metab. 26:118-124.

McAllister, J. M., B. Modi, B. A. Miller, J. Biegler, R. Bruggeman, R. S. Legro, and J. F. Strauss. 2014. Overexpression of a DENND1A isoform produces a polycystic ovary syndrome theca phenotype. Proc. Natl. Acad. Sci. USA 111:E1519-E1527.

McDaneld, T. G., L. A. Kuehn, M. G. Thomas, W. M. Snelling, T. P. Smith, E. J. Pollak, J. B. Cole, and J. W. Keele. 2014. Genomewide association study of reproductive efficiency in female cattle. J. Anim. Sci. 92:1945-1957.

Milazzotto, M. P., M. D. Goissis, J. L. Chitwood, K. Annes, C. A. Soares, J. Ispada, M. E. O. Á. Assumpção, and P. J. Ross. 2016. Early cleavages influence the molecular and the metabolic pattern of individually cultured bovine blastocysts. Mol. Reprod. Dev. $83: 324-336$

Minozzi, G., E. L. Nicolazzi, A. Stella, S. Biffani, R. Negrini, B. Lazzari, P. Ajmone-Marsan, and J. L. Williams. 2013. Genome wide analysis of fertility and production traits in Italian Holstein cattle. PLoS One 8:e80219.

Monniaux, D., C. Rico, H. Larroque, R. Dalbiès-Tran, C. Médigue, F. Clément, and S. Fabre. 2010. L'hormone antimüllérienne, prédicteur endocrinien de la réponse à une stimulation ovarienne chez les bovins. Gynecol. Obstet. Fertil. 38:465-470.

Moore, K., and W. W. Thatcher. 2006. Major advances associated with reproduction in dairy cattle. J. Dairy Sci. 89:1254-1266.

Needleman, P., J. Turk, B. A. Jakschik, A. R. Morrison, and J. B. Lefkowith. 1986. Arachidonic acid metabolism. Annu. Rev. Biochem. 55:69-102.

O'Shea, L. C., J. Mehta, P. Lonergan, C. Hensey, and T. Fair. 2012. Developmental competence in oocytes and cumulus cells: candidate genes and networks. Syst Biol Reprod Med 58:88-101.

Parker Gaddis, K. L., S. Dikmen, D. J. Null, J. B. Cole, and P. J. Hansen. 2017. Evaluation of genetic components in traits related to superovulation, in vitro fertilization, and embryo transfer in Holstein cattle. J. Dairy Sci. 100:2877-2891.

Parker Gaddis, K. L., D. J. Null, and J. B. Cole. 2016. Explorations in genome-wide association studies and network analyses with dairy cattle fertility traits. J. Dairy Sci. 99:6420-6435. 
Peluso, C., F. L. A. Fonseca, I. F. Rodart, V. Cavalcanti, G. Gastaldo, D. M. Christofolini, C. P. Barbosa, and B. Bianco. 2014. AMH: An ovarian reserve biomarker in assisted reproduction. Clin. Chim. Acta 437:175-182.

Rico, C., L. Drouilhet, P. Salvetti, R. Dalbiès-Tran, P. Jarrier, J.-L. Touzé, E. Pillet, C. Ponsart, S. Fabre, and D. Monniaux. 2012. Determination of anti-Müllerian hormone concentrations in blood as a tool to select Holstein donor cows for embryo production: from the laboratory to the farm. Reprod. Fertil. Dev. 24:932.

Rico, C., S. Fabre, C. Medigue, N. d. Clemente, F. Clement, M. Bontoux, J.-L. Touze, M. Dupont, E. Briant, B. Remy, J.-F. Beckers, and D. Monniaux. 2009. Anti-Mullerian hormone is an endocrine marker of ovarian gonadotropin-responsive follicles and can help to predict superovulatory responses in the cow. Biol. Reprod. 80:50-59.

Saint-Dizier, M., C. Guyader-Joly, G. Charpigny, B. Grimard, P. Humblot, and A. A. Ponter. 2011. Expression of enzymes involved in the synthesis of prostaglandin E2 in bovine in vitro-produced embryos. Zygote 19:277-283.

Sargolzaei, M. 2014. SNP1101 User's Guide. Version 1.0.

Sargolzaei, M., J. P. Chesnais, and F. S. Schenkel. 2014. A new approach for efficient genotype imputation using information from relatives. BMC Genomics 15:478.

Sargolzaei, M., F. S. Schenkel, G. B. Jansen, and L. R. Schaeffer. 2008. Extent of linkage disequilibrium in Holstein cattle in North America. J. Dairy Sci. 91:2106-2117.

Sharov, A. A., Y. Piao, R. Matoba, D. B. Dudekula, Y. Qian, V. VanBuren, G. Falco, P. R. Martin, C. A. Stagg, U. C. Bassey, Y. Wang, M. G. Carter, T. Hamatani, K. Aiba, H. Akutsu, L. Sharova, T. S. Tanaka, W. L. Kimber, T. Yoshikawa, S. A. Jaradat, S. Pantano, R. Nagaraja, K. R. Boheler, D. Taub, R. J. Hodes, D. L. Longo, D. Schlessinger, J. Keller, E. Klotz, G. Kelsoe, A. Umezawa, A. L. Vescovi, J. Rossant, T. Kunath, B. L. M. Hogan, A. Curci, M. D'Urso, J. Kelso, W. Hide, and M. S. H. Ko. 2003. Transcriptome analysis of mouse stem cells and early embryos. PLoS Biol. 1:E74.

Souza, A. H., P. D. Carvalho, A. E. Rozner, L. M. Vieira, K. S. Hackbart, R. W. Bender, A. R. Dresch, J. P. Verstegen, R. D. Shaver, and M. C. Wiltbank. 2015. Relationship between circulating antiMüllerian hormone (AMH) and superovulatory response of highproducing dairy cows. J. Dairy Sci. 98:169-178.

St John, J. C., J. Facucho-Oliveira, Y. Jiang, R. Kelly, and R. Salah. 2010. Mitochondrial DNA transmission, replication and inheritance: A journey from the gamete through the embryo and into offspring and embryonic stem cells. Hum. Reprod. Update 16:488 509.
Sugimoto, M., S. Sasaki, T. Watanabe, S. Nishimura, A. Ideta, M. Yamazaki, K. Matsuda, M. Yuzaki, K. Sakimura, Y. Aoyagi, and Y. Sugimoto. 2010. Ionotropic glutamate receptor AMPA 1 is associated with ovulation rate. PLoS One 5:e13817.

Thompson, J. G., R. J. Partridge, F. D. Houghton, C. I. Cox, and H. J. Leese. 1996. Oxygen uptake and carbohydrate metabolism by in vitro derived bovine embryos. J. Reprod. Fertil. 106:299-306.

Torres, A., M. Batista, P. Diniz, E. Silva, L. Mateus, and L. Lopes-daCosta. 2015. Effects of oocyte donor age and embryonic stage of development on transcription of genes coding for enzymes of the prostaglandins and progesterone synthesis pathways in bovine in vitro produced embryos. Zygote 23:802-812.

Triepels, R., L. van den Heuvel, J. Loeffen, R. Smeets, F. Trijbels, and J. Smeitink. 1998. The nuclear-encoded human NADH:ubiquinone oxidoreductase NDUFA8 subunit: cDNA cloning, chromosomal localization, tissue distribution, and mutation detection in complexI-deficient patients. Hum. Genet. 103:557-563.

VanRaden, P. M., and P. G. Sullivan. 2010. International genomic evaluation methods for dairy cattle. Genet. Sel. Evol. 42:7.

Wang, H., Y. Wen, S. Mooney, B. Behr, and M. L. Polan. 2002. Phospholipase A2 and cyclooxygenase gene expression in human preimplantation embryos. J. Clin. Endocrinol. Metab. 87:2629-2634.

Xiao, Z., J. Patrakka, M. Nukui, L. Chi, D. Niu, C. Betsholtz, T. Pikkarainan, S. Vainio, and K. Tryggvason. 2011. Deficiency in crumbs homolog 2 (Crb2) affects gastrulation and results in embryonic lethality in mice. Dev. Dyn. 240:2646-2656.

Yang, W.-C., S.-J. Li, K.-Q. Tang, G.-H. Hua, C.-Y. Zhang, J.-N. Yu, L. Han, and L.-G. Yang. 2010. Polymorphisms in the 5' upstream region of the FSH receptor gene, and their association with superovulation traits in Chinese Holstein cows. Anim. Reprod. Sci. 119:172-177.

Yang, W.-C., S.-J. Li, Y.-H. Xie, K.-Q. Tang, G.-H. Hua, C.-Y. Zhang, and L.-G. Yang. 2011. Two novel SNPs of the type I gonadotropin releasing hormone receptor gene and their associations with superovulation traits in Chinese Holstein cows. Livest. Sci. 136:164-168.

Yang, W.-C., L.-G. Yang, H. Riaz, K.-Q. Tang, L. Chen, and S.-J. Li. 2013. Effects in cattle of genetic variation within the IGF1R gene on the superovulation performance and pregnancy rates after embryo transfer. Anim. Reprod. Sci. 143:24-29.

Zhang, D., M. Li, W. Ma, Y. Hou, Y.-H. Li, S.-W. Li, Q.-Y. Sun, and W.-H. Wang. 2005. Localization of mitotic arrest deficient 1 (MAD1) in mouse oocytes during the first meiosis and its functions as a spindle checkpoint protein. Biol. Reprod. 72:58-68. 